\title{
Analysis and Calculation of Double Circular Arc Gear Meshing Impact Model
}

\author{
Li Yang ${ }^{*}, 1,2$, Wu Bao-lin ${ }^{1}$ and Zhu Lin-lin ${ }^{1}$ \\ ${ }^{I}$ Department of Mechanical Engineering, Tianjin Polytechnic University, Tianjin 300387 China \\ ${ }^{2}$ Department of Mechanical and Electrical Engineering, Qingdao Technological University (Lin Yi), Linyi 273400 \\ China
}

\begin{abstract}
The goal of this study is to propose a new theoretical approach for the analysis of the impact of the double circular arc gear meshing. The gear meshing impact dynamic model for four different meshing states was firstly built in the gear system. According to the mechanical dynamics and dynamics of the gear system, the reasons for the meshing impact and the impact type of double-circular-arc gear were analyzed in the paper. The content of the paper covers: (i) analysis of the mechanism of meshing impact; (ii) the practical meshing impact process; (iii) establishment of meshing impact model; (iv) solution of the meshing impact radius; (v) calculation of the meshing impact force in theory. The reverse method and the graphing method were used to determine the starting positions of impact and its coordinate formulas were built. Formula of impact velocity, impact force and impact radius were also established. Impact force is calculated with formulas constructed above. This paper's target is quite innovative and applicable and the paper gives a new way for double circular arc gear meshing impact research.
\end{abstract}

Keywords: Double circular arc gear, impact force, impact velocity, meshing impact model.

\section{INTRODUCTION}

The double circular arc gear drive is one kind of an axial conjugate gear drive meshing at points. Gear transmission depends on the axial movement along contact trace lines [1]. There are two contact trace lines in one gear teeth. Multiplepoint contact and multiple-tooth meshing is the basic meshing characteristic of the gear. Double circular arc gear has higher strength of bending and contact compared with involute gear for the same parameters. The gear has been widely used in industry machinery.

Because of the manufacturing errors and the deformation under the load, the practical contact point departsjavascript: $\operatorname{void}(0)$; from the theoretical meshing point, resulting in meshing impact when tooth surfaces are entering or exiting meshing (collectively referred to as 'meshing impact') in the meshing process. Meshing impact affects the working performance of the gear transmission system. Meshing impact generates noise and vibration and limits the gear's life.

Computer modeling, simulation and the static analysis by finite element method of the gear have been done by many researchers [2-4]. Some researchers have built double circular arc gear meshing impact models [5-7], but effects in different meshing states are not thoroughly studied.

The gear meshing impact dynamic model for four different meshing states was proposed with the reverse method and the graphing method. The starting positions of impact and its coordinate formulas were analyzed. Formula of impact velocity, impact force and impact radius were also established.

Tooth profile of the gear conforms to Chinese standard GB/T12759-1991 in this paper as shown in Fig. (1):

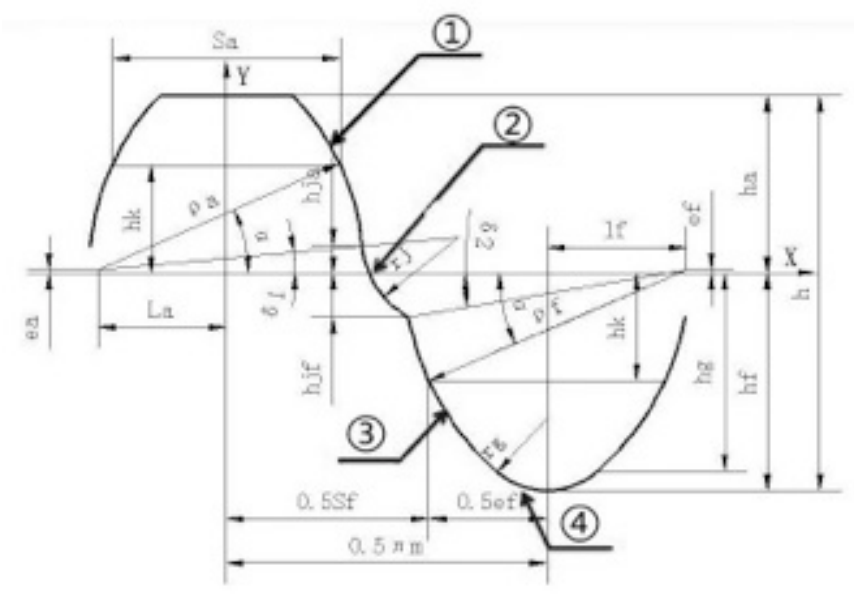

Fig. (1). Tooth profile of the gear.

A complete tooth profile composed of four circular arcs symmetrical around the $Y$-axis. The concave tooth is in the pitch circle. The convex tooth is out the pitch circle. The driving teeth maybe concave teeth or convex teeth. The axial pitches of the driving teeth ( $t_{1}$ for short) are maybe greater than or less than that of the driven teeth ( $t_{1}$ for short).

So the double circular arc gear meshing impact model can be classified into the following four situations: 
(1) The driving tooth is concave teeth and $t_{1}>t_{2}$.

(2) The driving tooth is concave teeth and $t_{1}<t_{2}$.

(3) The driving tooth is convex teeth and $t_{1}<t_{2}$.

(4) The driving tooth is convex teeth and $t_{1}>t_{2}$.

\section{MECHANISM AND PROCESS OF MESHING IMPACT}

\subsection{Meshing Impact Mechanism}

Axial pitch tolerance is denoted by $\Delta_{\alpha}$ and $\Delta_{\alpha}=t_{1}-t_{2}$. Because of manufacturing and assembling errors, $\Delta_{\alpha}$ makes the helical parameters of two gears change and subsequently changes the gear transmission ratio.

The formula of transmission ratio is:

$i=\frac{z_{2}}{z_{1}}=\frac{p_{2}}{p_{1}}$

Differentiate both sides of formula (1), we get:

$d(i)=i \bullet\left(\frac{d p_{2}}{p_{2}}-\frac{d p_{1}}{p_{1}}\right)$

The meshing impact mechanism of double circular arc gear is essentially the change of transmission ratio caused by the axial pitch tolerance.

\subsection{Meshing Impact Process}

Two types of meshing impact caused by axial pitch tolerance are taken as examples to show the meshing impact process.

Here is the meshing impact process when $t_{1}<t_{2}$ as shown in Fig. (2): Active tooth 3 will engage the driven tooth 4 ahead of time when the former gear pair, tooth 1 and tooth 2 , finish the meshing, which leads to meshing impact.

Here is the meshing impact process when $t_{1}>t_{2}$, as shown in Fig. (3): There is gap between tooth 3 and tooth 4, because $t_{1}>t_{2}$. When the former gear pair of tooth 1 and tooth 2 have finished meshing, tooth 1 will keep sliding on the tooth 2 until the gap diminishes.

\section{MODELING AND DISCUSSION}

The relationship between $\mathrm{u}$ and $u_{F}$ is as shown in Fig. (4). The equation of gear end dynamic load $u$ is:

$u=u_{F} \bullet \cos \beta$

The practical contact point P' departsjavascript:void(0); from the theoretical meshing point P. Velocity component along the gears end surfaces normal are unequal when impact occurs. The projections of the velocity along the gear end surface normal are respectively $v_{1}$ and $v_{2}$. The difference between $v_{1}$ and $v_{2}$ can be referred to as the impact velocity $v_{s}$, which can be formulated as:

$\begin{cases}v_{\mathrm{s}}=\left|v_{1}-v_{2}\right|=P E \bullet\left(\omega_{1}+\omega_{2}\right) & t_{1}<t_{2} \\ v_{\mathrm{s}}=\left|v_{1}-v_{2}\right|=P^{\prime} E \bullet\left(\omega_{1}+\omega_{2}\right) & t_{1}>t_{2}\end{cases}$ (a) Axial impact graph

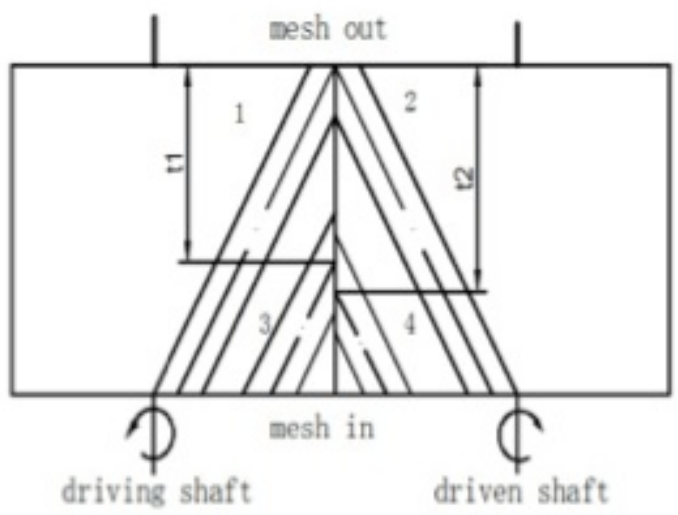

(b) Gear end surface impact graph

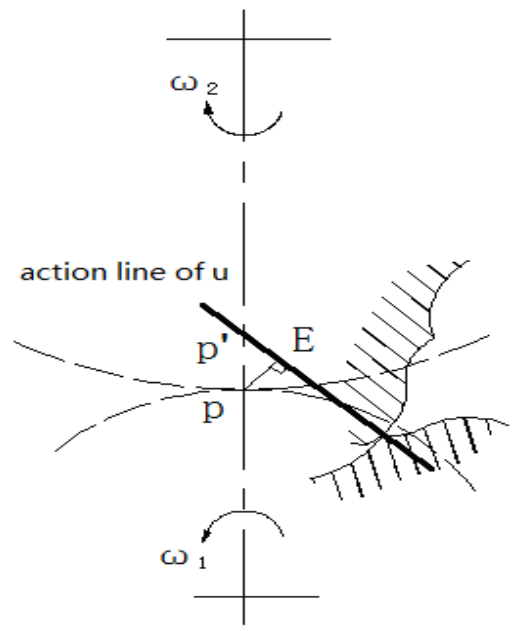

Fig. (2). Meshing impact graph when $t_{1}<t_{2}$.

The gear meshing system can be simplified into a torsion system without considering the elastic deformation of drive shaft, the supporting shaft and box, as shown in the following figure. By transforming the moment of inertia of the meshing gears into equivalent mass in action line, torque becomes force and moment of inertia becomes mass, as shown in Fig. (5).

$\left\{\begin{array}{l}\mathrm{m}_{\mathrm{eq} 1}=\frac{\mathrm{J}_{1}}{\left[\mathrm{n} \cdot R_{1} \cdot \cos (\alpha)\right]^{2}} \\ \mathrm{~m}_{\mathrm{eq} 2}=\frac{\mathrm{J}_{2}}{\left[\mathrm{n} \cdot R_{2} \cdot \cos (\alpha)\right]^{2}} \\ \mathrm{~m}_{\varepsilon}=\frac{\mathrm{m}_{\mathrm{eq} 1} \mathrm{~m}_{\mathrm{eq} 2}}{\mathrm{~m}_{\mathrm{eq} 1}+\mathrm{m}_{\mathrm{eq} 2}}\end{array}\right.$

We should take into account the number of meshing points, $n$. We assume $\mathrm{n}=1$ in the derivation process, $S_{1}$ and $S_{2}$ is the distance in action line, $P$ is the driving force, $W$ is the resistance and the dynamic load is the impact force in the model.

The tooth is an elastic body and its deformation $\delta$ is measured by $\delta=S_{1}-S_{2}$. The maximum deformation is denoted by $\delta_{\max }$. 
(a) Axial impact graph

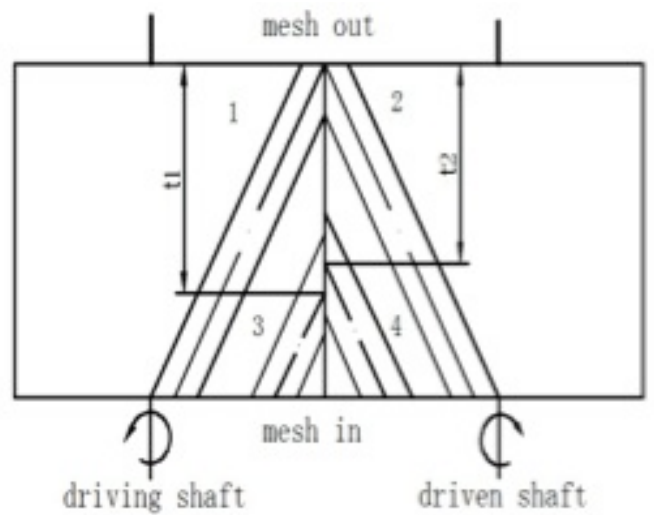

(b) Gear end surface impact graph

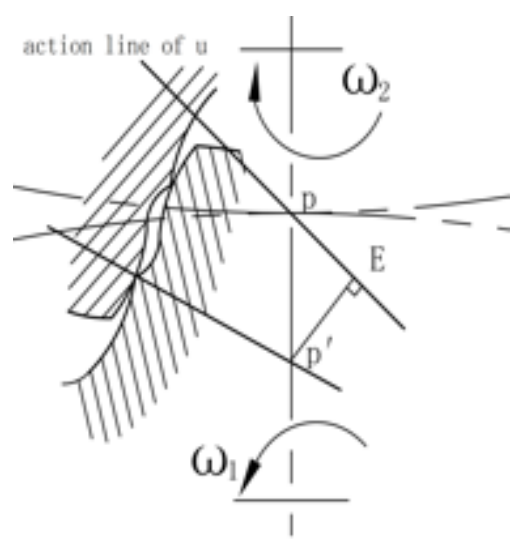

Fig. (3). Meshing impact graph when $t_{1}>t_{2}$.

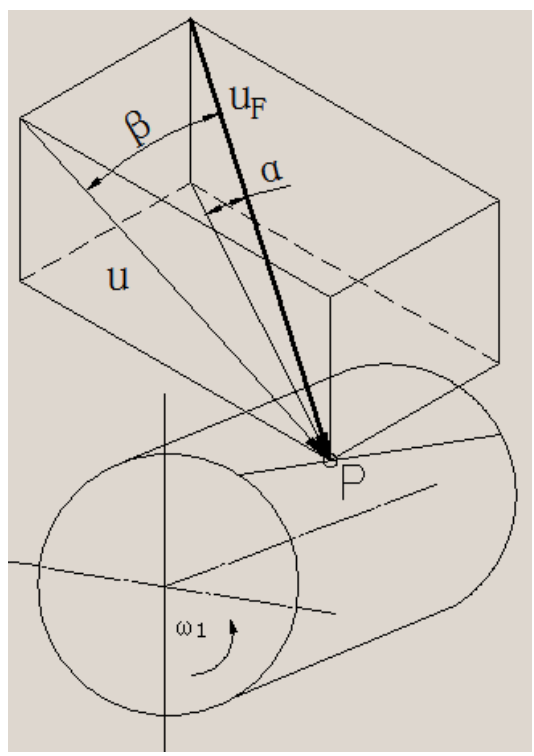

Fig. (4). The relationship between $\mathrm{u}$ and $u_{F}$.

Impact velocity $\mathrm{v}_{s}$ generates impact kinetic energy $E_{\mathrm{v} s}$. The impact kinetic energy $E_{v s}$ transforms into deformation energy $U$ based on the law of conservation of energy until impact velocity $\mathrm{v}_{s}$ diminishes to zero.

$$
\left\{\begin{array}{l}
\mathrm{E}_{v \mathrm{~s}}=\frac{1}{2} \frac{\mathrm{m}_{\text {eq } 1} \mathrm{~m}_{\text {eq2 }}}{\left(\mathrm{m}_{\mathrm{eq} 1}+\mathrm{m}_{\mathrm{eq} 2}\right)} v_{\mathrm{s}}^{2}=\frac{1}{2} \frac{\mathrm{J}_{1} \mathrm{~J}_{2}}{\left(\mathrm{~J}_{1} \mathrm{R}_{2}^{2}+\mathrm{J}_{2} \mathrm{R}_{1}^{2}\right) n} v_{\mathrm{s}}^{2} \\
\mathrm{U}=\frac{1}{2} \mathrm{~K} \delta_{\text {max }}{ }^{2}=\frac{\mathrm{F}_{\mathrm{S}}{ }^{2}}{2 \mathrm{~K}} \\
\mathrm{E}_{v \mathrm{~s}}=\mathrm{U}
\end{array}\right.
$$

The result of solution is:

$u=F_{s}=v_{s} \frac{1}{\cos \alpha} \sqrt{\frac{K}{n} \frac{J_{1} J_{2}}{\left(J_{1} R_{2}^{2}+J_{2} R_{1}^{2}\right)}}$

And the parameters in formula (7) are formulated as:

$\left\{\begin{array}{l}R_{1}=\frac{m_{n} \bullet z_{1}}{2 \cos (\beta)} ; R_{2}=\frac{m_{n} \bullet z_{2}}{2 \cos (\beta)} \\ D_{1}=2 \bullet R_{1} ; D_{2}=2 \bullet R_{2} \\ J_{1}=\frac{\pi \rho D_{1}^{4} B}{32} ; J_{2}=\frac{\pi \rho D_{2}^{4} B}{32}\end{array}\right.$

We developed different models for solving impact radius in 4 different meshing situations as follows.

(a) Rotation model

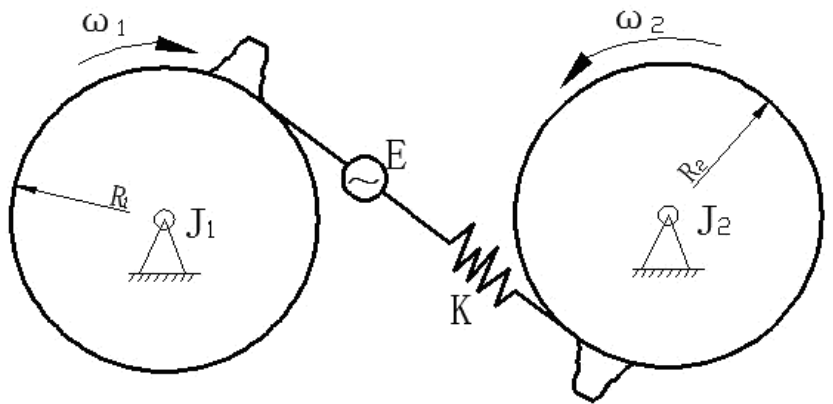

(b) Linear model

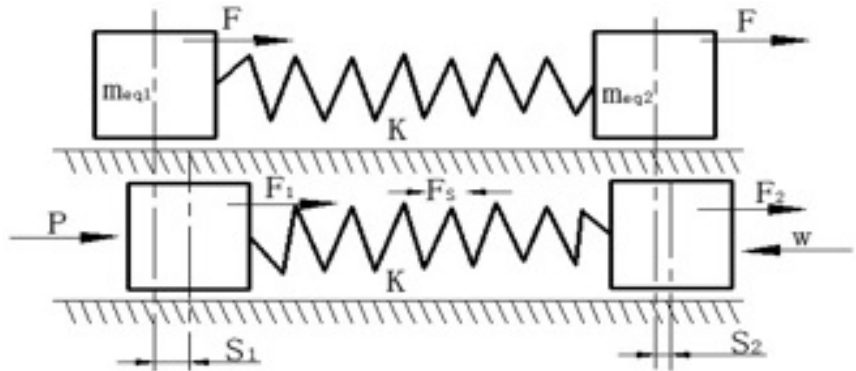

Fig. (5). Meshing model.

\subsection{Solving for Meshing Impact Radius}

The curvature radius of convex tooth profile tends to be equal to concave tooth profile in the running-in process. Suppose that the contact arc radius is $\rho_{A V}$ and $\rho_{A V}=\left(\rho_{\alpha}+\rho_{f}\right) / 2$. 
We can draw a conclusion from formula (4) that impact radius $P E$ or $P^{\prime} E$ can determine impact velocity $v_{s}$, with given angular velocity $\omega_{1}$ and $\omega_{2}$ for driving and driven gears. So it is necessary to build a model to ascertain the solution of impact radius $P E$ or $P^{\prime} E$.

\subsubsection{The Concave Tooth Meshing Impact Radius When $t_{1}<t_{2}$}

As shown in Fig. (6), $\mathrm{P}$ is the theoretical meshing position for meshing gear pair of driving gear 1' and driven gear 2'. However, because of $t_{1}<t_{2}$, the practical contact

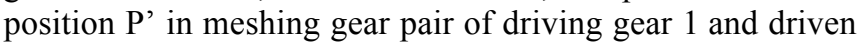
gear 2 departsjavascript:void(0); from the theoretical meshing point $\mathrm{P}$, as shown in Fig. (6).

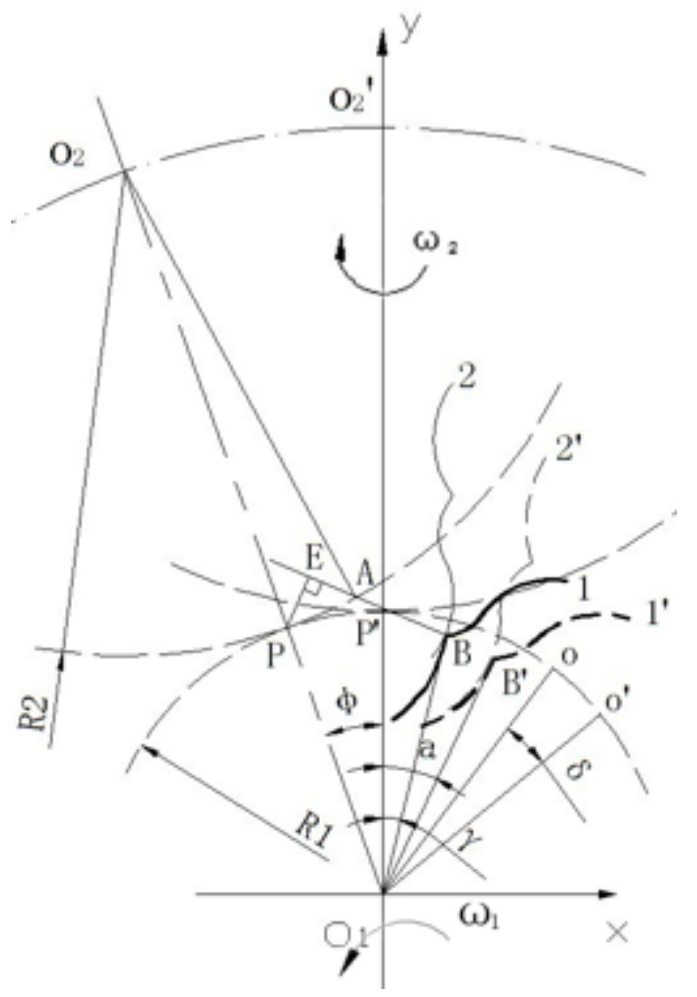

Fig. (6). Meshing impact position.

The reverse method is: Fix the driving gear 1, reversely roll the driven gear 2 without slipping and with angular velocity of $\left(\omega_{1}+\omega_{2}\right)$ until gear 2 meshes with gear 1 in the point $B$ on the dedendum. The point $B$ is the start position of the impact. The impact position and impact radius can be calculated by the reverse method and the graphing method.

The coordinates of points $A$ and $B$ are:

$$
\left\{\begin{array}{l}
x_{A}=-\left(R_{1}+R_{2}\right) \sin \phi+R_{2} \sin \left(\phi+\frac{\phi}{i}\right) \\
y_{A}=\left(R_{1}+R_{2}\right) \cos \phi-R_{2} \cos \left(\phi+\frac{\phi}{i}\right) \\
x_{B}=\left(R_{1}-h_{j f}\right) \sin \gamma ; \\
y_{B}=\left(R_{1}-h_{i f}\right) \cos \gamma
\end{array}\right.
$$

The meaning and calculation of other parameters $\alpha, \delta, \varphi$, and $\lambda$ refer to Fig. (6), and the formula (10):

$\left\{\begin{array}{l}\gamma=\alpha-\delta \\ a=\arccos \left(\frac{R_{1}^{2}+\left(R_{1}-h_{i f}\right)^{2}-\rho^{2}}{2 R_{1}\left(R_{1}-h_{j f}\right)}\right) \\ \delta=\frac{\Delta}{R_{1}} \\ \Delta=\Delta_{\alpha} \tan \beta\end{array}\right.$

The contact arc $r$ adius $\rho_{A V}$ is the distance between point $\mathrm{A}$ and point $\mathrm{B}$ based on the geometric structure characteristics of double circular arc gear.

$$
|A B|=\sqrt{\left(x_{A}-x_{B}\right)^{2}+\left(y_{A}-y_{B}\right)^{2}}=\rho_{A V}
$$
(10):

Dag the coordinate of points A and B into the formula

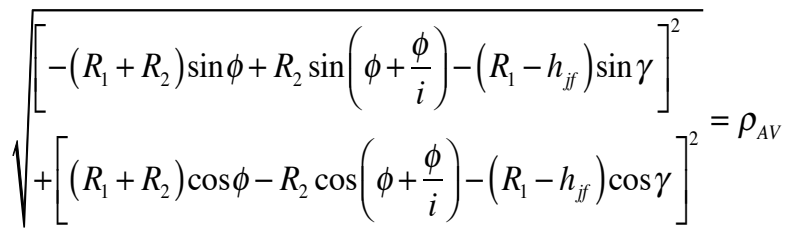

Coordinate of the instantaneous meshing nodes $P$ is:

$x_{p}=-R_{1} \sin \phi ; y_{P}=R_{1} \cos \phi$

The vertical length $\mathrm{PE}$ is the impact radius and the linear equation of $\mathrm{PE}$ are:

$\frac{x-x_{A}}{x_{B}-x_{A}}=\frac{y-y_{A}}{y_{B}-y_{A}}$

The distance from $\mathrm{P}$ to $\mathrm{E}$ is:

$|P E|=\left|\frac{a_{1} x_{P}-b_{1} y_{P}+b_{1} y_{B}-a_{1} x_{B}}{c_{1}}\right|$

In which,

$\left\{\begin{array}{l}a_{1}=\left(R_{1}+R_{2}\right) \cos \phi-R_{2} \cos \left(\phi+\frac{\phi}{i}\right)-\left(R_{1}-h_{i f}\right) \cos \gamma \\ b_{1}=-\left(R_{1}+R_{2}\right) \sin \phi+R_{2} \sin \left(\phi+\frac{\phi}{i}\right)-\left(R_{1}-h_{j f}\right) \sin \gamma \\ c_{1}=\sqrt{a_{1}^{2}+b_{1}^{2}}\end{array}\right.$

When the transmission parameters and pitch circle surface error $\Delta$ is confirmed, we can obtain the specific coordinate value of points $\mathrm{A}, \mathrm{B}$ and $\mathrm{P}$.

Impact radius PE can be calculated from formula (15). The impact velocity $v_{s}$ can be calculated from formula (4).

\subsubsection{The Concave Tooth Meshing Impact Radius when $t_{1}>t_{2}$}

The concave tooth meshing impact radius when $t_{1}>t_{2}$ is as shown in Fig. (7). Using the same derivation method to solve impact radius $\mathrm{P}$ 'E, 
$\left\{\begin{array}{l}x_{A}=-R_{2} \sin \phi_{2} ; x_{B}=\left(R_{1}-h_{j f}\right) \sin \gamma \\ y_{A}=\left(R_{1}+R_{2}\right)-R_{2} \cos \phi_{2} ; y_{B}=\left(R_{1}-h_{j f}\right) \cos \gamma\end{array}\right.$

$\sqrt{\frac{\left[R_{2} \sin \phi_{2}+\left(R_{1}-h_{j f}\right) \sin \gamma\right]^{2}+}{\left[\left(R_{1}+R_{2}\right)-R_{2} \cos \phi_{2}-\left(R_{1}-h_{j f}\right) \cos \gamma\right]^{2}}}=\rho_{A V}$

$\left\{\begin{array}{l}\alpha=\arccos \left(\frac{R_{1}^{2}+\left(R_{1}-h_{k}\right)^{2}-\rho^{2}}{2 R_{1}\left(R_{1}-h_{k}\right)}\right) ; \phi_{2}=\frac{\phi_{1}}{i} \\ \Delta=\Delta_{\alpha} \tan \beta ; \delta=\frac{\Delta}{R_{1}} ; \gamma=\alpha-\phi_{1}-\delta\end{array}\right.$

$\left\{\begin{array}{l}x_{C}=-R_{1} \sin \left(\delta+\phi_{1}\right) ; y_{C}=R_{1} \cos \left(\delta+\phi_{1}\right) \\ x_{p^{\prime}}=0 ; y_{p^{\prime}}=-\frac{y_{B}-y_{C}}{x_{B}-x_{C}} \times x_{C}+y_{C}\end{array}\right.$

Coordinates of A, B, C and P' are calculated by formula (17-20). The point $B$ is the start position of the impact. Impact radius $\mathrm{P}$ ' $\mathrm{E}$ and the impact velocity $v_{s}$ can also be calculated by formula (15) and formula (4).

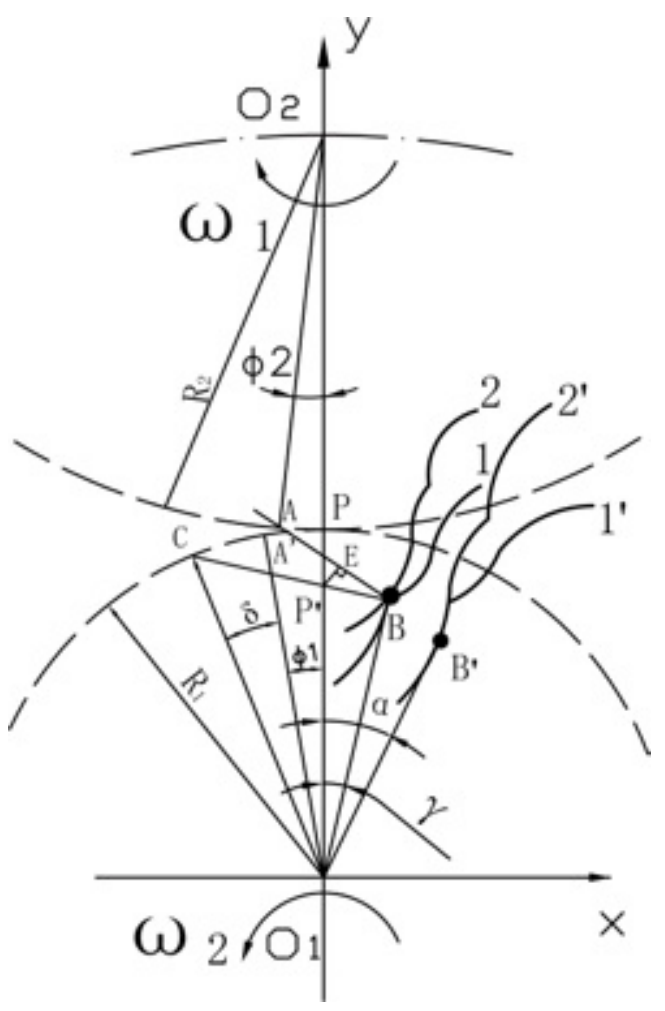

Fig. (7). Meshing impact position

\subsubsection{The convex tooth meshing impact radius when $t_{1}<t_{2}$}

The point $\mathrm{A}$ is the start position of the impact. The active convex tooth arc center is represented symbolically by $\mathrm{O}$ in Fig. (8).

Similarly, the formulae are:
$\left\{\begin{array}{l}x_{A}=-\left(R_{1}+R_{2}\right) \sin \varphi+\left(R_{2}-h_{i f}\right) \sin \left(\varphi+\frac{\varphi}{i}+\tau\right) \\ y_{A}=\left(R_{1}+R_{2}\right) \cos \varphi-\left(R_{2}-h_{i f}\right) \cos \left(\varphi+\frac{\varphi}{i}+\tau\right) \\ x_{O}=R_{1} \sin \gamma ; y_{O}=R_{1} \cos \gamma\end{array}\right.$

$\left\{\begin{array}{l}\theta=\arccos \left(\frac{R_{2}^{2}+\left(R_{2}-h_{i f}\right)^{2}-\rho^{2}}{2 R_{2}\left(R_{2}-h_{i f}\right)}\right) ; \tau=\arccos \left(\frac{R_{2}^{2}+\left(R_{2}-h_{i f}\right)^{2}-l_{\tau}^{2}}{2 R_{2}\left(R_{2}-h_{i f f}\right)}\right) \\ l_{\tau}=\sqrt{r_{j}^{2}+r_{j}^{2}-2 r_{j} r_{j} \cos \left(\arcsin \left(\frac{\left(r+r_{j}\right) \sin \delta_{1}+h_{i f}}{r_{j}}\right)-\arcsin \left(\frac{\left(r+r_{j}\right) \sin \delta_{1}}{r_{j}}\right)\right.}\end{array}\right.$

$\alpha=(\theta+\tau) i ; \gamma=\alpha-\delta ; \delta=\frac{\Delta}{R_{1}}$

Symbols in the above formulae have the same meaning with the foregoing formulae. Symbol $r_{j}$ represents radius of the connecting arc. Other parameters refer to Fig. (8).

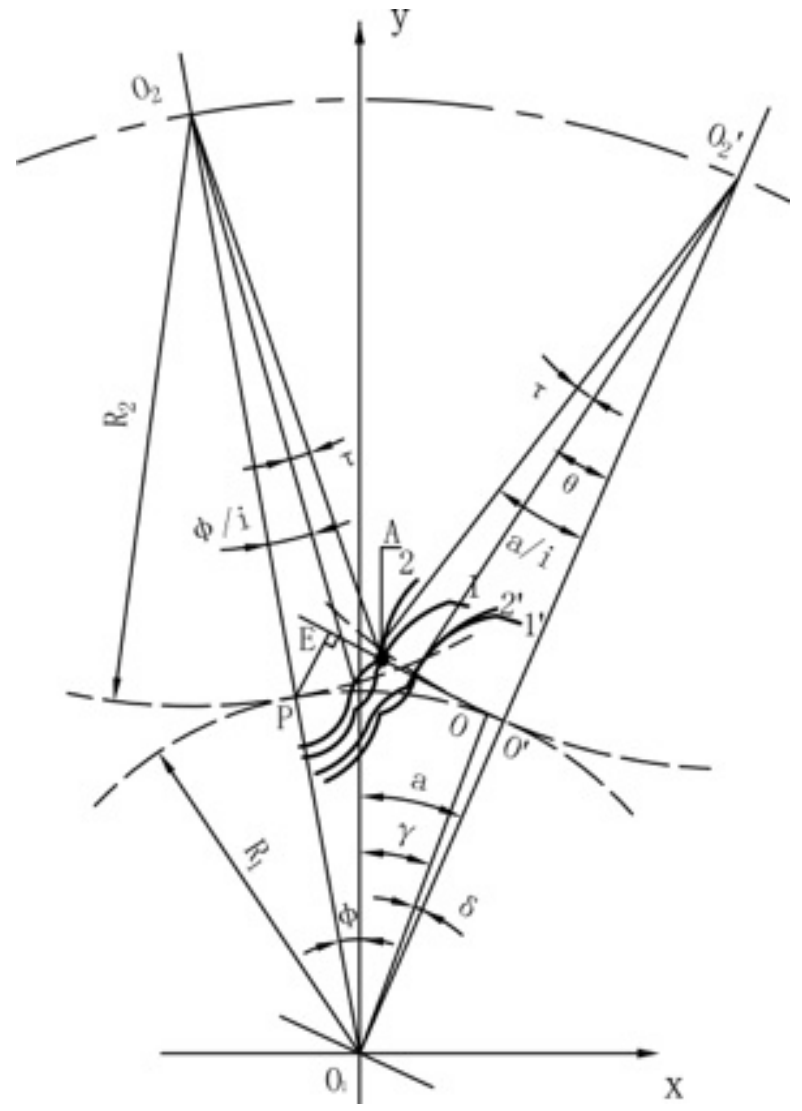

Fig. (8). Meshing impact position.

Again, impact radius $P E$ and the impact velocity $v_{s}$ can be calculated by formula (15) and formula (4).

\subsubsection{The Convex Tooth Meshing Impact Radius when $t_{1}>t_{2}$}

The convex tooth meshing impact radius when $t_{1}>t_{2}$ is as shown in Fig. (9). Point $\mathrm{A}$ is the start position of the 
impact. New coordinates of A, B and P' are calculated as the following formulae shows:

$$
\begin{aligned}
& \left\{\begin{array}{l}
x_{A}=R_{1} \sin \varphi_{1} ; y_{A}=R_{1} \cos \varphi_{1}-R_{1} \\
x_{B}=\left(R_{2}-h_{i f}\right) \sin \left(\theta+\phi_{2}\right) ; y_{B}=R_{2}-\left(R_{2}-h_{i f}\right) \cos \left(\theta+\phi_{2}\right)
\end{array}\right. \\
& \theta=\arccos \left(\frac{R_{2}^{2}+\left(R_{2}-h_{j f}\right)^{2}-\rho^{2}}{2 R_{2}\left(R_{2}-h_{j f}\right)}\right)
\end{aligned}
$$

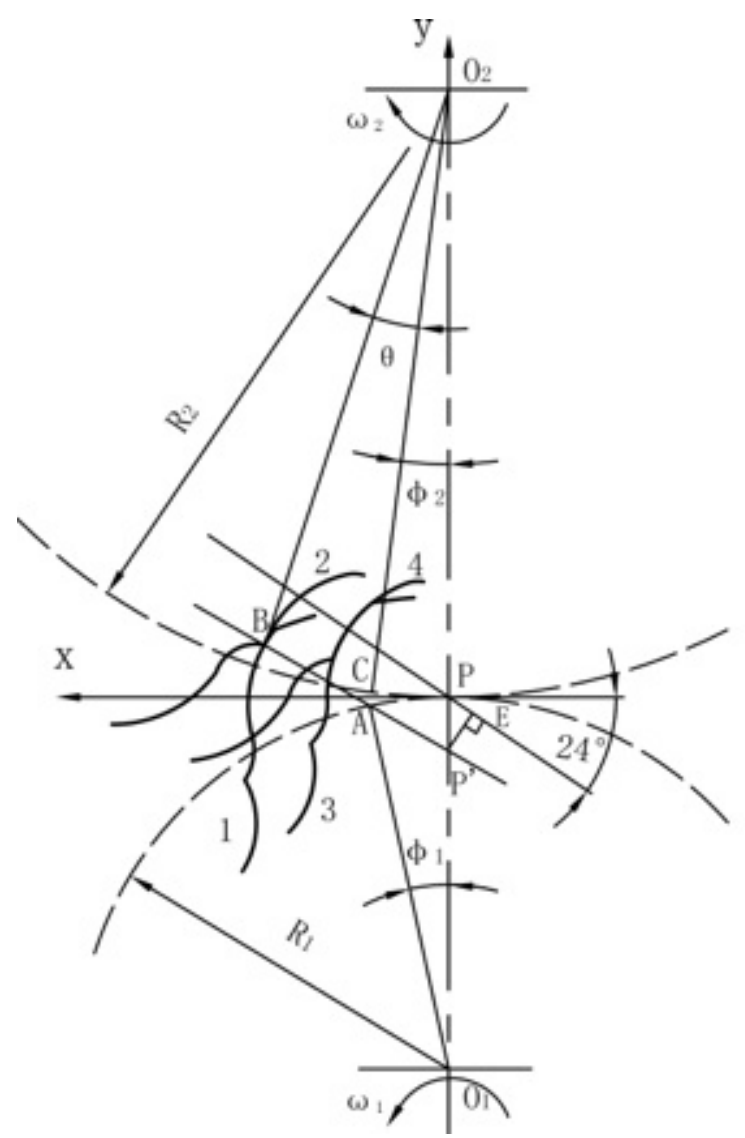

Fig. (9). Meshing impact position.

The Y-coordinate of point $\mathrm{P}$ ' is:

$Y_{P^{\prime}}=y_{A}+\frac{y_{A}-y_{B}}{x_{A}-x_{B}} x_{A}$

Impact radius P'E can be calculated by:

$P^{\prime} E=\left|Y_{P^{\prime}}\right| \bullet \cos \left(24^{\circ}\right)$

The impact velocity $v_{s}$ is calculated by formula (4).

\subsection{Calculation of the Meshing Impact Force}

The impact velocity $v_{s}$ can be calculated from formulae above-deduced. The calculation of stiffness consults ref. [7, 8].

The numerical model of the gear is used in this research with the following design parameters: Normal module $m_{n}=4 \mathrm{~mm}$; Number of gears teeth $z_{1}=z_{2}=24$; Face width $B=55 \mathrm{~mm}$; Helix angle $\beta=15^{\circ}$; Pitch circle surface error
$\Delta=0.1 \mathrm{~mm}$; the rotational speed of the driving gear $n_{1}=200 \mathrm{r} / \mathrm{min}$; Driving torque $T=100 \mathrm{~N} / \mathrm{m}$; Gear material density $\rho=7850 \times 10^{9} \mathrm{~kg} / \mathrm{mm}^{3}$.

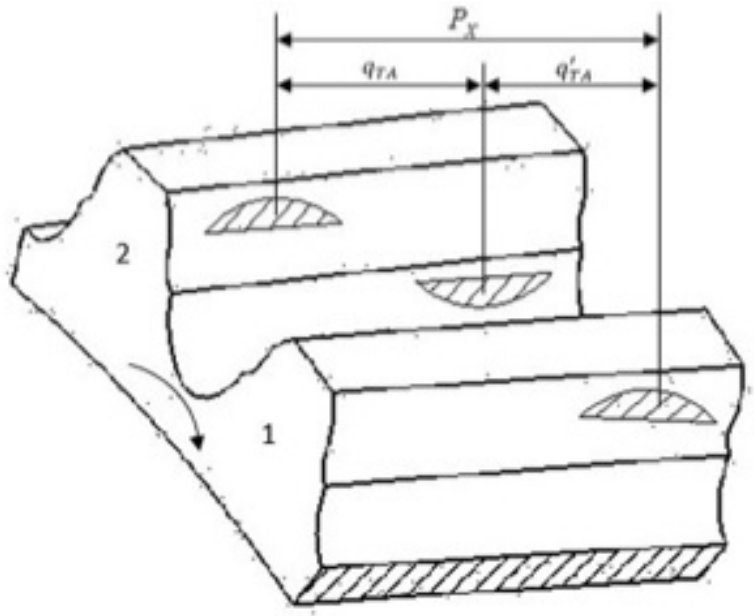

Fig. (10). Contact position on tooth surfaces.

Contact points are spread over an area under the load. Parameters in Fig. (10) are defined as:

$\left\{\begin{array}{l}P_{x}=\frac{\pi \bullet m_{n}}{\sin (\beta)} ; \Delta b=B-P_{x} \\ q_{T A}=\frac{0.5 \pi m_{n}-0.5 j+2 l_{a}+2 e_{a} \bullet \cot a}{\sin \beta}-2\left(\rho_{a}+\frac{e_{a}}{\sin a}\right) \cos a \sin \beta \\ q_{T A}^{\prime}=P_{x}-q_{T A}\end{array}\right.$

where, $P_{x}$ is gear axial pitch ; $q_{T A}$ is the distance between the contact point on convex surface and the contact point on concave surface in the same tooth; $q_{T A}^{\prime}$ is the distance between the contact point on concave surface and the contact point on convex surface in neighboring tooth; $j$ is the gear backlash, and $j=0.06 m_{n}$.

The following results can be obtained based on the data above: $P_{x}=48.55 \mathrm{~mm} ; q_{T A}=41.85 \mathrm{~mm} ; q_{T A}^{\prime}=6.70 \mathrm{~mm}$; $\Delta b=6.45 \mathrm{~mm}$;

We can conclude the inequality:

$\Delta b<P_{x}-q_{T A}$

The meshing figures when $\Delta b<P_{x}-q_{T A}$ is shown as in Fig. (11):

The meshing cycle is equal with gear axial pitch $P_{x}$. We take 2\#convex surface as research from 2-2 to 4-4 area. Two mesh impact states of mesh in or mesh out process are analyzed in this paper, namely zone A and zone E. There are three meshing points in the two mesh states of $\mathrm{A}$ zone and $\mathrm{E}$ zone, so meshing points $n=3$.

Based on the parameters and formulae above, calculation of the meshing impact force $F_{s}$ is just as Tables $\mathbf{1}$ and $\mathbf{2}$ shows: 
Table 1. Calculation of the meshing impact force $F_{s}$ when $t_{1}<t_{2}$

\begin{tabular}{|c|c|c|c|c|}
\hline \multirow{2}{*}{ Calculation Parameters } & \multicolumn{2}{|c|}{ Concave Tooth Impact } & \multicolumn{2}{|c|}{ Convex Tooth Impact } \\
\cline { 2 - 5 } & Mesh In & Mesh Out & Mesh In & Mesh Out \\
\hline \hline Meshing stiffness $K(\mathrm{~N} / \mathrm{mm})$ & $1.8084 \times 10^{6}$ & $7.685 \times 10^{5}$ & $1.771 \times 10^{6}$ & $7.1088 \times 10^{5}$ \\
\hline Impact velocity $V_{s}(\mathrm{~mm} / \mathrm{s})$ & 61.92 & 61.92 & 36.33 & 36.33 \\
\hline Impact force $F_{s}(\mathrm{~N})$ & $4.816 \times 10^{4}$ & $3.1392 \times 10^{4}$ & $2.796 \times 10^{4}$ & $1.7715 \times 10^{4}$ \\
\hline
\end{tabular}

Table 2. Calculation of the meshing impact force $F_{s}$ when $t_{1}<t_{2}$.

\begin{tabular}{|c|c|c|c|c|}
\hline \multirow{2}{*}{ Calculation Parameters } & \multicolumn{2}{|c|}{ Concave Tooth Impact } & \multicolumn{2}{c|}{ Convex Tooth Impact } \\
\cline { 2 - 5 } & Mesh In & Mesh Out & Mesh In & Mesh Out \\
\hline \hline Meshing stiffness $K(\mathrm{~N} / \mathrm{mm})$ & $1.8084 \times 10^{6}$ & $7.685 \times 10^{5}$ & $6.771 \times 10^{6}$ & $7.1088 \times 10^{5}$ \\
\hline Impact velocity $V_{s}(\mathrm{~mm} / \mathrm{s})$ & 81.57 & 81.57 & 60.01 & 60.01 \\
\hline Impact force $F_{s}(\mathrm{~N})$ & $6.344 \times 10^{4}$ & $4.1354 \times 10^{4}$ & $4.6185 \times 10^{4}$ & $2.9261 \times 10^{4}$ \\
\hline
\end{tabular}

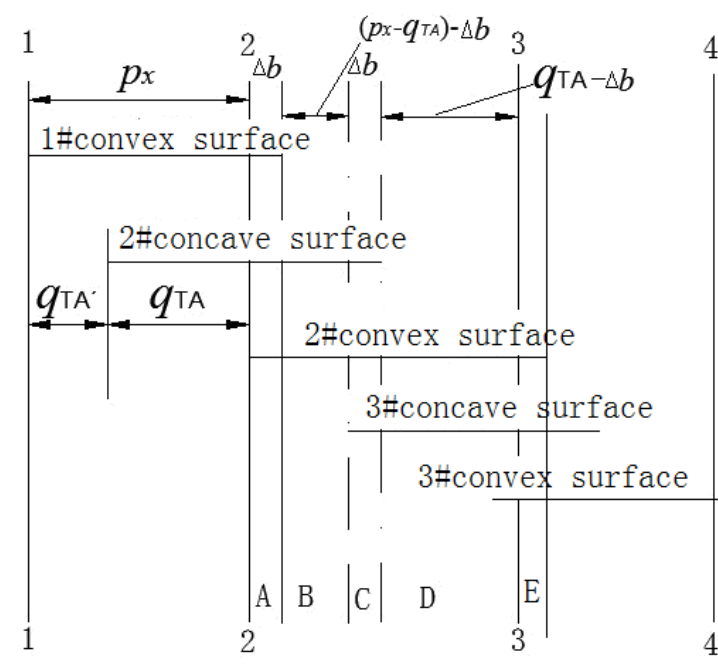

Fig. (11). Meshing figure when $\Delta b<P_{x}-q_{T A}$.

Several assumptions were made from the calculation above: (1) Impact velocity when gears come into mesh equals impact velocity when gears come out of mesh, on the premise that Pitch circle surface error $\Delta$ does not change; (2) Meshing stiffness $K$ will not change if the gear meshes in the same mesh point.

\section{CONCLUSION}

(1) The change of transmission ratio is caused by axial pitch tolerance. There are 4 mesh impact situations in the double circular arc gear meshing impact model.

(2) With the reverse method and the graphing method, the gear meshing impact dynamic model was firstly built. The starting positions of impact are determined and their coordinate formulas are built. Formula of impact velocity, impact force and impact radius were also established. Impact force is calculated with formulas constructed above.
(3) Impact force $F_{s}$ is dependent on impact velocity $V_{s}$, meshing stiffness $\mathrm{K}$, gear moment of inertia $J_{i}$ pressure angle $\alpha$ and other gear parameters. Impact radius $P E$ or $P^{\prime} E$ determine impact velocity $V_{s}$, which is only in connection with pitch circle surface error $\Delta$.

(4) Results show Concave tooth impact is greater than convex tooth impact; Mesh impact when $t_{1}>t_{2}$ is greater than mesh impact when $t_{1}<t_{2}$.

These results illustrated with analyses a new way for double circular arc gear meshing impact research.

\section{CONFLICT OF INTEREST}

The authors confirm that this article content has no conflict of interest.

\section{ACKNOWLEDGEMENTS}

Declared none.

\section{APPENDIX}

Definitions of variables in this paper are shown in the follow nomenclature:

$t_{i}$ : The axial pitch for the driving tooth $(\mathrm{i}=1)$ or for the driven tooth $(\mathrm{i}=2)$.

$i$ : Transmission ratio;

$z_{i}$ : Driving gear tooth number $(\mathrm{i}=1)$ or driven gear tooth number $(\mathrm{i}=2)$;

$p_{i}$ : Driving gear helical parameter $(\mathrm{i}=1)$ or driven gear helical parameter $(i=2)$.

$u_{F}$ : Dynamic load normal line; 
$u$ : The gear end projection of $u_{F}$;

$\beta$ : Helical angle.

$\Delta_{\alpha}:$ Axial pitch tolerance, and $\Delta_{\alpha}=t_{1}-t_{2}$.

$v_{s}:$ Impact velocity.

$\mathrm{F}_{s}:$ Impact force.

$P E$ and $P^{\prime} E$ : Impact radius which is the distance between theoretical meshing point and practical line of force $\mathrm{u},(P E$ when $t_{1}<t_{2}$ or P'E when $t_{1}>t_{2}$ );

$\omega_{i}$ : Angular velocity for driving gear $(i=1)$ and driven gear $(i=2)$.

$J_{i}$ : Driving gear moment of inertia $(i=1)$ or driven gear moment of inertia $(i=2)$;

$m_{\text {eqi }}$ : Driving gear equivalent mass $(i=1)$ or driven gear equivalent mass $(i=2)$;

$m_{\varepsilon}:$ System equivalent.

$S_{1}, S_{2}$ : Distance in action line.

$P$ : Driving force.

$W:$ Resistance force.

$\delta:$ Mesh deformation.

$\delta_{\max }:$ The maximum deformation.

$E_{v s}:$ Impact kinetic energy.

$U$ : Deformation energy.

$u$ : Dynamic load is impact force in the model, that is $u=\mathrm{F}_{s}$.

$n$ : The number of meshing points.

$K$ : Meshing stiffness;

$B$ : Tooth width;

$\alpha$ : Pressure angle and $\alpha=24^{\circ}$.

$m_{n}$ : Normal module. $\rho$ : Gear material density.

$R_{i}$ : Pitch circle radius for driving gear $(i=1)$ and driven gear $(i=2)$.

$\rho_{A V}:$ The contact arc radius and $\rho_{A V}=\left(\rho_{\alpha}+\rho_{f}\right) / 2$.

$\rho_{\alpha}$ : The curvature radius of convex tooth profile.

$\rho_{f}$ : The curvature radius of concave tooth profile.

$h_{i f}$ : Concave tooth connection point high.

$\Delta:$ Pitch circle surface error.

$h k$ : The distance between contact point with pitch line, and $h k=0.545 * m_{n}$.

$r_{j}$ : Radius of the connecting arc.

\section{REFERENCES}

[1] J. Shao, Double Circular Arc Gear, Beijing: China Machine, Press, 1994.

[2] F. Zhang, and H. Liu, "The Calculation and Analysis on the Meshing Impact of Gear Transmission", Mechanical Science and Technology for Aerospace Engineering, vol.31, no.5, pp.718-722, 2012.

[3] Z. Chen, and Y. Shao, "Dynamic simulation of spur gear with tooth root crack propagating along tooth width and crack depth", Engineering Failure Analysis, vol.18, no.8, pp. 939-946, 2011.

[4] F. L. Litvin, A. Fuentes, I. Gonzalez-Perez, L. Carnevali, and T. M. Sep, "New version of Novikov-Wildhaber helical gears: computerized design, simulation of meshing and stress analysis", Computer Methods in Applied Mechanics and Engineering, vol.191, no. 49-50, pp. 5707-5740, 2002.

[5] S. Chen, J. Tang, and L. Wu, "Dynamics analysis of a crowned gear transmission system with impact damping: Based on experimental transmission error", Mechanism and Machine Theory, vol. 74, pp. 31-47, 2014

[6] B. Wu, S. Yang, and J. Yao, "Theoretical analysis on meshing impact of involute gears", Mechanical Science and Technology, vol.22, no.1, pp.55-57, 2003.

[7] G. Ye, and X. Ye, "A new method for seeking the optimum gear tooth profiles - the theoretical basis of Wildhaber-Novikov gearing", Mechanism and Machine Theory, vol.37, no.10, pp.1087$1103,2002$.

[8] B. Wu, H. Meng, and J. Shao, "Vibration analysis of a doublecircular-arc helical gearing system", Chinese Journal of Mechanical Engineering, vol. 35, no. 5, pp. 85-89, 1999. 